\title{
Facteurs de variation et prédiction de la dégradabilité de l'azote des fourrages verts dans le rumen
}

\author{
P Le Goffe 1, R Vérité 2, P Faverdin 2 \\ 1 ENSAR, 65, rue de Saint-Brieuc, 35042 Rennes Cedex; \\ 2 INRA, station de recherches sur la vache laitière, 35590 Saint-Gilles, France
}

(Reçu le $1^{\text {er }}$ octobre 1991; accepté le 7 septembre 1992)

\begin{abstract}
Résumé - Les objectifs de ce travail étaient d'expliquer les variations de la dégradabilité de l'azote (N) des fourrages verts par celles de leur composition chimique et de leurs conditions de croissance, et d'élaborer des équations de prédiction de la proportion de $\mathrm{N}$ dégradable dans le rumen. La dégradabilité de $\mathrm{N}$, mesurée en sachets de nylon et in vitro (solubilité, fermentescibilité, dégradation enzymatique pendant $1 \mathrm{~h}=\mathrm{dE} 1$ ), ainsi que la composition chimique (matière sèche, matières azotées totales = MAT, NDF, ADF, lignine, $\mathrm{N}$ des parois) et les conditions de croissance (température moyenne) ont été déterminées chez 72 fourrages verts lyophilisés. Les fourrages, différant par le stade végétatif, l'espèce, la saison, la fertilisation azotée, le lieu de récolte et les conditions climatiques ont été récoltés entre le 3 avril 1985 et le 15 octobre 1986. Dans la population complète, la dégradabilité théorique de $N$ en sachets de nylon (DTN avec un taux de passage de $0,06 \mathrm{~h}^{-1}$ ) varie de 65 à $90 \%$; elle est avant tout liée négativement à la température et aux teneurs en parois. Dans une population homogène quant au cycle, à la température et la teneur en parois, elle augmente avec la teneur en MAT. La prédiction de la DTN est possible à partir de la composition chimique (MAT pour les graminées de $1^{\text {er }}$ cycle; MAT + NDF pour les autres fourrages), mais la précision est très améliorée lorsque la $\mathrm{dE} 1$ de $\mathrm{N}$ est introduite dans les équations. Les limites de notre travail, liées à la méthode des sachets (conditionnement des fourrages, contamination microbienne) et au transit ruminal des fourrages verts sont discutées.
\end{abstract}

fourrage vert / azote / rumen / dégradabilité / sachet / prédiction

Summary - Variation factors and prediction of herbage nitrogen degradability in the rumen. The objectives of this study were: 1) to explain the variations in rumen degradability of herbage nitrogen $(N)$ by the changes in chemical composition and growth conditions; 2) to provide equations to predict the proportion of degradable $N$ in the rumen. Feed $N$ degradability in nylon bag and in vitro (solubility, microbial fermentation, enzymatic degradation over $1 \mathrm{~h}=E d_{1}$ ), chemical composition (dry matter, crude protein $=C P, N D F, A D F$, lignin, fibre $N$ ) and growth conditions (temperature) were measured in 72 freeze-dried herbages. The herbages at different stages of maturity of different species, seasons, $N$ fertilizations, areas and climatic conditions, were harvested between April 31985 and October 15 1986. In the whole population, effective rumen degradability of feed $N$ in situ (EPD with outflow rate of $0.06 \mathrm{~h}^{-1}$ ) was negatively related to temperature and fibre content; in a population 
of the same cut, temperature and fibre content, EPD increased with CP. EPD prediction was possible using only the chemical composition (CP, grasses first cut; $N D F+C P$, other herbages); however, precision was improved when $E d$, was introduced in the equations. The limits of the study, related to the nylon bag method (herbage processing, microbial contamination) and herbage rumen transit have been discussed.

herbage / nitrogen / rumen / degradability / nylon bag / prediction

\section{INTRODUCTION}

En dépit de leurs teneurs en matières azotées (MAT) souvent élevées, les fourrages verts distribués seuls ne permettent que des apports protéiques intestinaux modérés, inférieurs aux quantités ingérées, et susceptibles de limiter la production des animaux en croissance ou en lactation (Thomson, 1982). C'est ainsi que la complémentation des fourrages verts à partir de sources azotées peu dégradables ou protégées est souvent plus efficace que la complémentation énergétique (Thomson, 1982; Beever, 1982). Les mesures in vivo du flux d'azote ( $N$ ) duodénal et de sa fraction d'origine microbienne sont imprécises et relativement peu nombreuses sur fourrages verts. II semble que la valeur azotée moyenne des fourrages verts ait pour origine une dégradation de $\mathbf{N}$ dans le rumen importante en moyenne, responsable de faibles apports protéiques alimentaires, bien que le rendement de la synthèse microbienne soit plutôt élevé (Beever, 1982; Demarquilly et Jarrige, 1982). Seule la phase de dégradation dans le rumen a été étudiée dans le travail présenté ici, en utilisant essentiellement la méthode des sachets de nylon.

Nous avons consacré des articles antérieurs (Le Goffe, 1988; Le Goffe et al, 1989; Le Goffe et Vérité, 1992; Le Goffe et al, 1993) à l'étude séparée des différents facteurs de variation de la dégradabilité de $\mathrm{N}$ des fourrages verts dans le rumen (stade de développement, fertilisation azotée, saison, espèce). Le présent travail a consisté à regrouper ces études, dans le but d'expliquer les variations de la dégradabilité par celles de la composition chimique et des conditions de croissance.

Sur un plan plus appliqué, il s'agissait d'élaborer des équations de prédiction de la proportion de $\mathrm{N}$ dégradable dans le rumen, paramètre indispensable à tout système d'expression de la valeur azotée des aliments pour ruminants (Alderman, 1987). Ces équations devaient permettre de : 1) constituer des tables de valeur de fourrages types; 2) déterminer rapidement et simplement la valeur d'un fourrage précis pour son utilisation zootechnique.

\section{MATÉRIEL ET MÉTHODES}

\section{Matériel végétal}

Soixante-douze fourrages verts, provenant pour la plupart du regroupement de 4 études, ont été récoltés entre le 3 avril 1985 et le 15 octobre 1986) (tableau I). Au total, la population comprend 27 ray-grass, 26 fétuques élevées, 10 trèfles violets, 5 luzernes et 4 dactyles; elle se divise en 35 fourrages du premier cycle et 37 repousses récoltées majoritairement en été. Plus de $80 \%$ des fourrages ont été récoltés à un stade précoce. Les conditions de croissance ont été caractérisées par la température et le rayonnement moyens pendant la croissance (Le Goffe et al, 1993). 
Tableau l. Les 4 études sur les facteurs de variation de la dégradabilité de l'azote des fourrages verts dans le rumen.

\begin{tabular}{|c|c|c|c|c|c|}
\hline Étude & Espèces & $\begin{array}{l}\text { Facteurs croisés } \\
\text { avec l'espèce }\end{array}$ & Lieu & Année & $\begin{array}{l}\text { Nombre de } \\
\text { fourrages }\end{array}$ \\
\hline Le Goffe, 1988 & $\begin{array}{l}\text { Ray-grass anglais } \\
\text { Trèfle violet }\end{array}$ & Stade au $y^{\text {er cycle }}$ & Rennes & 1985 & $13^{*}$ \\
\hline Le Goffe et al, 1989 & Fétuque élevée & $\begin{array}{l}\text { Fertilisation azotée } \\
\text { Rang du cycle } \\
\text { Âge au } 4^{\theta} \text { cycle }\end{array}$ & Lusignan & 1986 & 18 \\
\hline Le Goffe et Vérité, 1992 & Ray-grass anglais & Rang du cycle & Rennes & 1986 & 6 \\
\hline Le Goffe et al, 1993 & $\begin{array}{l}\text { Ray-grass anglais } \\
\text { Ray-grass italien } \\
\text { Ray-grass hybride } \\
\text { Fétuque élevée } \\
\text { Dactyle } \\
\text { Trèfle violet } \\
\text { Luzeme }\end{array}$ & Saison & Rennes & $\begin{array}{l}1985 \\
1986\end{array}$ & 31 \\
\hline
\end{tabular}

- Quatre stades supplémentaires du 1er cycle d'un ray-grass anglais ont été récoltés en 1986.

Les échantillons de fourrage sont pour la majorité congelés à $-35^{\circ} \mathrm{C}$ dès la récolte, stockés à $-15^{\circ} \mathrm{C}$, puis lyophilisés et broyés à la grille de $1,5 \mathrm{~mm}$ pour la technique in situ. Cependant, les 24 fourrages du printemps 1985 ont été broyés à la grille de $0,8 \mathrm{~mm}$, et parmi eux, 12 fourrages ont été congelés dans l'azote liquide. La dégrabilité des fourrages verts est cependant peu affectée par ces modifications du conditionnement (Le Goffe, 1991).

\section{Analyses chimiques}

Les teneurs en matière sèche (MS), MAT, NDF (Van Soest et Wine, 1967), ADF et lignine (Van Soest, 1963), azote du NDF et de l'ADF ont été déterminées, comme indiqué chez le Goffe et al (1993). La composition chimique moyenne des fourrages étudiés est donnée au tableau II.

Tableau II. Moyenne et variabilité de la composition chimique de 72 fourrages verts.

\begin{tabular}{lrllllll}
\hline Statistique & $\begin{array}{l}\text { MS } \\
(\%)\end{array}$ & $\begin{array}{l}\text { MAT } \\
(\% m s)\end{array}$ & $\begin{array}{l}\text { NDF } \\
(\% m s)\end{array}$ & $\begin{array}{l}\text { ADF } \\
(\% m s)\end{array}$ & $\begin{array}{l}\text { Lignine } \\
(\% m s)\end{array}$ & $\begin{array}{l}\text { N-NDF* } \\
(\% n t)\end{array}$ & $\begin{array}{l}\text { N-ADF* } \\
\text { (\%int) }\end{array}$ \\
\hline Moyenne & 18,1 & 18,9 & 46,2 & 24,8 & 2,3 & 15,1 & 2,3 \\
Écart type & 3,6 & 4,3 & 9,6 & 4,3 & 1,1 & 5,2 & 1,0 \\
Minimum & 9,8 & 7,7 & 23,9 & 13,1 & 1,0 & 5,4 & 0,9 \\
Maximum & 25,9 & 28,4 & 65,7 & 35,2 & 6,7 & 28,4 & 4,9 \\
\hline
\end{tabular}

* Azote lié au NDF/ADF, en pourcentage de l'azote total. 


\section{Mesures de dégradabilité}

Trois tests de dégradabilité de $\mathrm{N}$ en laboratoire ont été réalisés : solubilité $(S)$ et fermentescibilité (F) in vitro (Vérité et Demarquilly, 1978); dégradation enzymatique pendant $1 \mathrm{~h}$ (dE1 : Aufrère et Cartailler, 1988). La mesure de la dégradation de MS et de $\mathrm{N}$ en sachets de nylon incubés $2,4,7,10,24$, et $48 \mathrm{~h}$ dans le rumen, et le calcul des dégradabilités théoriques de MS (DTMS) et de N (DTN) ont été effectués comme précédemment (Le Goffe et al, 1993). Le taux de passage des particules hors du rumen a été pris à $0,06 h^{-1}$. La moyenne et la dispersion des mesures de dégradabilité sur la population sont présentées dans le tableau III.

\section{Méthodologie statistique}

L'analyse factorielle multiple (AFM; Escofier et Pagès, 1988) a été utilisée pour étudier les relations entre dégradabilité et caractéristiques intrinsèques des fourrages verts. L'AFM convient particulièrement pour traiter des tableaux individus $x$ variables où les variables sont structurées en groupes homogènes. Le programme utilisé est issu du logiciel ADDAD (1989). Les résultats présentés concernent essentiellement l'analyse de la population complète ( 72 fourrages verts); cependant, une $2^{\mathrm{e}}$ analyse a été réalisée sur les seules graminées. Les variables actives sont divisées en 4 groupes : dégradabilité de MS, de $\mathrm{N}$, conditions de croissance, composition chimique (tableau IV).
Les relations de prédiction de la DTN ont étẻ élaborées par régression progressive descendante.

\section{RÉSULTATS}

\section{Relations entre dégradabilité et caractéristiques intrinsèques des fourrages}

l'AFM sur la population complète révèle une direction prépondérante ( $46 \%$ de l'inertie), commune aux 4 groupes actifs qui participent à parts égales à sa construction. Les relations entre variables sont synthétisées sur le plan des deux premiers axes factoriels qui cumule $61 \%$ de l'inertie totale (fig 1). L'importance de la dégradabilité de MS et $N$, quel que soit le paramètre considéré (dégradation surtout jusqu'à 24 $h$, fractions a, vitesses $c$, DTMS et DTN, S, $\mathrm{F}, \mathrm{N}$ non lié aux parois), apparaît sur le premier axe. La dégradabilité est opposée à la température et au rayonnement moyens, qui sont eux-mêmes associés aux teneurs en NDF et MS, et, dans une moindre mesure, aux autres fractions pariétales (ADF, lignine). Cependant, à dégradabilité de la MS donnée, la dégradabi-

Tableau III. Moyenne et dispersion de la dégradabilité de la matière sèche et de l'azote de 72 fourrages verts dans le rumen (en pourcentage).

\begin{tabular}{lrrrrrrrrrr}
\hline Statistique & ams & bms & cms & DTMS $S$ & $F$ & an & bn & $c n$ & DTN \\
\hline Moyenne & 41,4 & 49,1 & 10,5 & 70,6 & 47,9 & 47,9 & 52,1 & 42,5 & 15,9 & 81,7 \\
Écart type & 6,5 & 6,9 & 5,5 & 7,3 & 6,6 & 9,6 & 12,2 & 12,5 & 7,1 & 5,3 \\
Minimum & 29,6 & 32,0 & 2,7 & 57,4 & 33,7 & 24,0 & 28,4 & 14,5 & 5,8 & 65,5 \\
Maximum & 57,5 & 62,9 & 29,3 & 85,2 & 61,4 & 70,5 & 79,0 & 69,0 & 37,5 & 89,8 \\
\hline
\end{tabular}

$\mathrm{S}$ : solubilité de $\mathrm{N}$ en salive artificielle; $\mathrm{F}$ : fermentescibilité in vitro de $\mathrm{N}$; ams, bms, cms (an, bn, cn) : paramètres du modèle décrivant la cinétique de dégradation de la MS (N) en sachets; DTMS (DTN) : dégradabilité théorique de la MS (N) en sachets $\left(k=0,06 \mathrm{~h}^{-1}\right)$. 
Tableau IV. Nature et composition des groupes de variables pris en compte dans l'AFM sur 72 fourrages verts.

\begin{tabular}{|c|c|c|c|}
\hline $\begin{array}{l}\text { Dégradabilité de } \\
\text { la matière sèche } \\
\text { (\%)* }\end{array}$ & $\begin{array}{l}\text { Dégradabilité } \\
\text { de l'azote } \\
(\%)^{*}\end{array}$ & $\begin{array}{l}\text { Conditions } \\
\text { de croissance }\end{array}$ & $\begin{array}{l}\text { Composition } \\
\text { chimique }\end{array}$ \\
\hline $\begin{array}{l}\text { Cinétique } \\
\text { ams, bms, cms } \\
\text { DTMS }\end{array}$ & $\begin{array}{l}\text { Cinétique } \\
\text { an, bn, cn } \\
\text { DTN } \\
\text { S } \\
\text { F }\end{array}$ & $\begin{array}{l}\text { Température }\left({ }^{\circ} \mathrm{C}\right) \\
\text { Rayonnement }\left(\mathrm{mj} / \mathrm{m}^{2}\right)\end{array}$ & $\begin{array}{l}\text { MS (\%) } \\
\text { MAT (\%ms) } \\
\text { NDF (\%ms) } \\
\text { ADF (\%ms) } \\
\text { lignine (\%ms) } \\
\text { N-NDS (\%nt) } \\
\text { N-ADS (\%nt) }\end{array}$ \\
\hline
\end{tabular}

- Cinétique MS et $\mathrm{N}$ : dégradation de MS et $\mathrm{N}$ en sachets à $2,4,7,10,24,48 \mathrm{~h}$; ams, bms, cms (an, bn, cn) : paramètres du modèle appliqué à la cinétique de MS (N); DTMS (DTN) : dégradabilité thèorique de la MS (N) en sachets $\left(k=0,06 \mathrm{~h}^{-1}\right) ; S=$ solubilité de $\mathrm{N}$ en salive artificielle; $F=$ fermentescibilité in vitro de $N$; ** $N$-NDS (ADS) : azote soluble dans le détergent neutre (acide), en pourcentage de l'azote total.

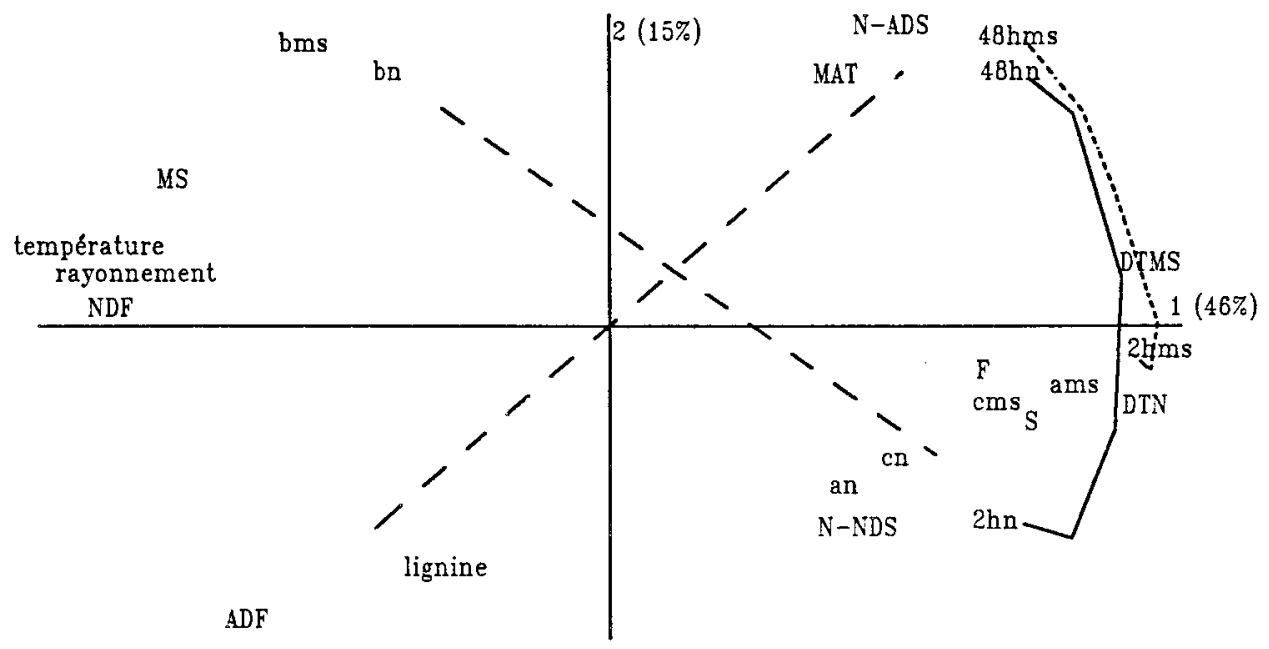

Fig 1. Relations entre dégradabilité, composition chimique et conditions de croissance des fourrages verts (Deux premiers axes de l'AFM sur 72 fourrages. $N$-NDS, $N$-ADS : $\% N$ total soluble dans les détergents neutre et acide; $S$ : solubilité de $N ; F$ : fermentescibilité in vitro de $N ; 48 \mathrm{~h} M S / \mathrm{N}$ : dégradation de MS/N à $48 \mathrm{~h} ; \mathrm{a}, \mathrm{b}, \mathrm{c}$, paramètres du modèle exponentiel; DTMS/DTN = dégradabilité théorique de $M S / N, k=0,06 \mathrm{~h}^{-1}$ ). 
lité de $\mathbf{N}$ de la luzerne est supérieure à celle des graminées, et plus encore à celle du trèfle violet (exemple de la DTN, fig 2). Le caractère commun de l'axe 1 aux 4 groupes actifs suggère que les variations de la dégradabilité, dues aux différents facteurs (espèce, saison, etc), sont susceptibles d'être expliquées en utilisant les mêmes critères chimiques ou climatiques. II y a également une opposition entre les critères de dégradabilité potentielle (dégradation à $48 \mathrm{~h}, \mathrm{~N}$ soluble dans le détergent acide) associés à la teneur en MAT, et le caractère lignifié d'un fourrage (ADF, lignine). Cet axe ainsi dégagé est perpendiculaire à un autre axe fictif, qui oppose les fractions lentement dégradables aux fractions rapidement dégradables et aux vitesses de dégradation. II semble donc exister une certaine indépendance entre les caractères de dégradabilité potentielle et de rapidité de dégradation chez les fourrages verts.

Les critères de dégradation en sachets sont surtout liés aux teneurs en parois. Quel que soit le constituant considéré (MS ou $N$ ), la dégradation en début d'incubation, le taux horaire de dégradation et la DT ont la meilleure liaison avec la teneur en NDF, tandis que la dégradation en fin d'incubation est davantage liée à la teneur en ADF et en lignine (tableau V). Les liaisons relatives à la MS sont cependant meilleures que celles relatives à $N$, notamment parce qu'elles rassemblent mieux graminées et légumineuses. C'est ainsi que la teneur en NDF est un mauvais prédicteur de la dégradation de $\mathrm{N}$ en début d'incubation : à NDF donné, elle est faible pour le trèfle violet et élevée au contraire pour les 1ers cycles à tiges de graminées (fig 3). La plupart des critères de dégradabilité de $\mathrm{N}$ in vitro ou en sachets sont peu liés à la teneur en MAT. La valeur de la corrélation entre la dégradation de $\mathrm{N}$ en sachets et la teneur en MAT augmente de 2 à $24 \mathrm{~h}$ et devient notable

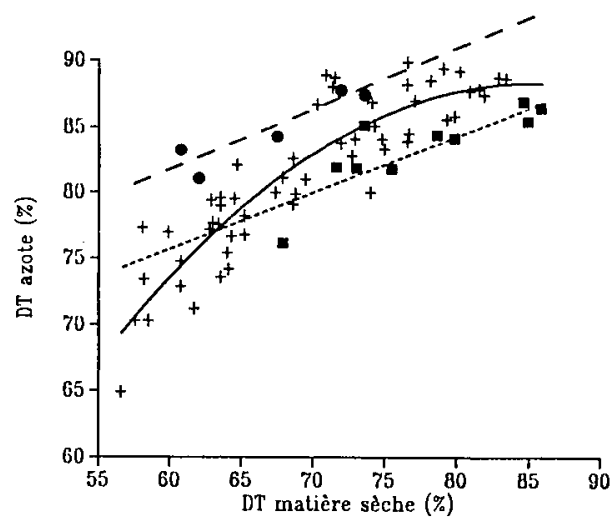

Fig 2. Relations entre les dégradabilités théoriques de $\mathrm{N}$ et MS $\left(k=0,06 \mathrm{~h}^{-1}\right) ; 72$ fourrages verts. + : graminées; DTN : $-80,6+3,97$ DTMS $-0,0233 \mathrm{DTMS}^{2} ; \boldsymbol{F}^{R}=0,82 ; \mathbf{E}:$ trèfle violet : $D T N=49,7+0,43$ DTMS; $\not P=0,73$; $\bullet$ : luzerne : DTN = 54,0+0,46 DTMS; $\not R=0,85$ ).

seulement à 24 h et 48 h $(0,67$ et 0,66 respectivement).

Sur la population de graminées, les associations de variables qui constituent les 2 directions principales de variation sont assez proches de celles observées sur la population totale. Les 2 modifications principales, sont : 1) l'apparition de la teneur en lignine, nettement opposée à la dégradabilité; 2) l'indépendance de la fraction immédiatement dégradée et de la vitesse de dégradation de la fraction lentement dégradable.

\section{Prédiction de la DTN}

Considérés isolément, le stade, la saison et l'espèce ont chacun en effet moyen compris entre 5 et 10 points de DTN (moyenne population $82 \%$ ); les variations dues à la fertilisation azotée et au lieu sont plus variables et encore plus modestes. Cependant, les différents facteurs se cu- 
Tableau V. Corrélations entre dégradabilité en sachets de la matière sèche et de l'azote et les teneurs en constituants pariétaux ( 72 fourrages verts).

\begin{tabular}{lllllllllllll} 
Matière sèche * & $d m s 2$ & $d m s 4$ & $d m s 7$ & $d m s 10$ & $d m s 24$ & $d m s 48$ & $a m s$ & $b m s$ & $c m s$ & DTMS \\
\hline NDF & $-0,81$ & $-0,82$ & $-0,79$ & $-0,82$ & $-0,61$ & $-0,37$ & $-0,66$ & $+0,54$ & $-0,67$ & $-0,78$ \\
ADF & $-0,63$ & $-0,58$ & $-0,64$ & $-0,65$ & $-0,75$ & $-0,78$ & $-0,47$ & $-0,06$ & $-0,36$ & $-0,71$ \\
lignine & $-0,20$ & $-0,12$ & $-0,18$ & $-0,21$ & $-0,52$ & $-0,80$ & $-0,25$ & $-0,42$ & $+0,27$ & $-0,32$ \\
& & & & & & & & & & \\
Azote * & $d n 2$ & $d n 4$ & $d n 7$ & $d n 10$ & $d n 24$ & $d n 48$ & an & bn & $c n$ & DTN \\
& & & & & & & & & & & \\
NDF & $-0,42$ & $-0,58$ & $-0,69$ & $-0,68$ & $-0,54$ & $-0,44$ & $-0,10$ & $+0,04$ & $-0,59$ & $-0,62$ \\
ADF & $-0,05$ & $-0,14$ & $-0,33$ & $-0,43$ & $-0,74$ & $-0,75$ & $+0,14$ & $-0,29$ & $-0,17$ & $-0,35$ \\
lignine & $-0,16$ & $-0,05$ & $-0,07$ & $-0,13$ & $-0,36$ & $-0,45$ & $-0,27$ & $+0,14$ & $+0,41$ & $-0,30$
\end{tabular}

- dms2 (dn2) : dégradation de la MS (N) à $2 \mathrm{~h}$; ams, bms, cms (an, bn, cn) = paramètres du modèle appliqué à la cinétique de la MS (N); DTMS (N) : dégradabilité théorique de la MS (N), $k=0,06 \mathrm{~h}^{-1}$.

mulent et la DTN varie de 65 à $90 \%$ sur la population.

En raison de la remontée de la DTN en septembre, il n'a pas été possible d'utiliser la date pour prédire la DTN sur les populations regroupant plusieurs cycles. En revanche, la date s'est avérée être un très

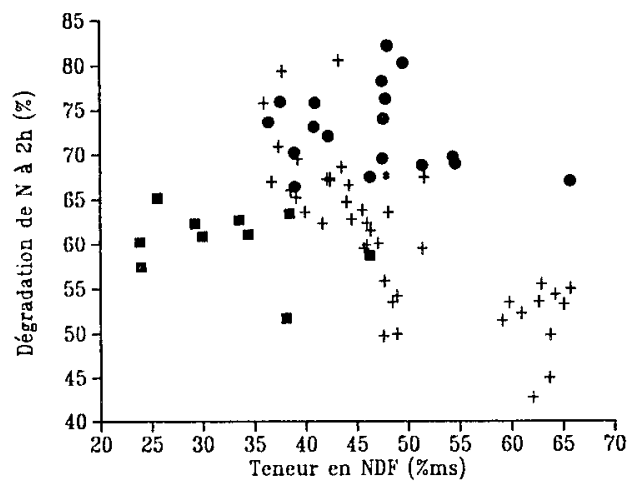

Fig 3. Relation entre la dégradation in situ de $\mathbf{N}$ à $2 \mathrm{~h}$ et la teneur en NDF; 72 fourrages verts; $1^{\text {er }}$ cycle à tiges de graminées; $\boldsymbol{U}$ : trèfle violet; + : autres. bon prédicteur de la DTN des premiers cycles de graminées (tableau VI).

Les constituants pariétaux sont les caractéristiques chimiques les plus liées à la DTN sur des populations regroupant plusieurs cycles. Chez les graminées, la teneur en lignine permet une meilleure prédiction que le NDF et surtout l'ADF. La teneur en MS, qui évolue dans le même sens que la température et la teneur en NDF, est également un prédicteur possible de la DTN des graminées, mais moins précis que les teneurs en constituants pariétaux (tableau VII). La teneur en MAT présente une faible liaison avec la DTN sur la population complète des 72 fourrages; cependant, en association avec les parois, elle devient significative lorsque le premier cycle et les repousses sont distingués chez les graminées (tableau VII). La DTN semble augmenter davantage avec la teneur en MAT chez les légumineuses $(0,9$ à 1,4 pts/pt) que chez les repousses de graminées $(0,7$ à $0,9 \mathrm{pt} / \mathrm{pt})$. Cependant, l'équation de prédiction de la DTN à partir 
Tableau VI. Prédiction de la dégradabilité théorique de l'azote (en pourcentage, $k=0,06 \mathrm{~h}^{-1}$ ) des graminées au premier cycle $(n=18 ; s i=3,7)$.

\begin{tabular}{llrrrr}
\hline Prédictrices & $a$ & $b 1$ & $b 2$ & $s r$ & $R^{2}$ \\
\hline Date $^{2}$ & 90,9 & $-0,0029$ & & 1,6 & 0,82 \\
MAT, MAT $^{2}$ & 58,4 & 3,0000 & $-0,075$ & 1,6 & 0,83 \\
\hline
\end{tabular}

si ou sr : écart type initial ou résiduel; date : jours écoulés depuis le $1^{\text {er }}$ avril.

Tableau VII. Prédiction de la dégradabilité théorique de l'azote des fourrages verts (en pourcentage, $k$ $=0,06 \mathbf{h}^{-1}$ ).

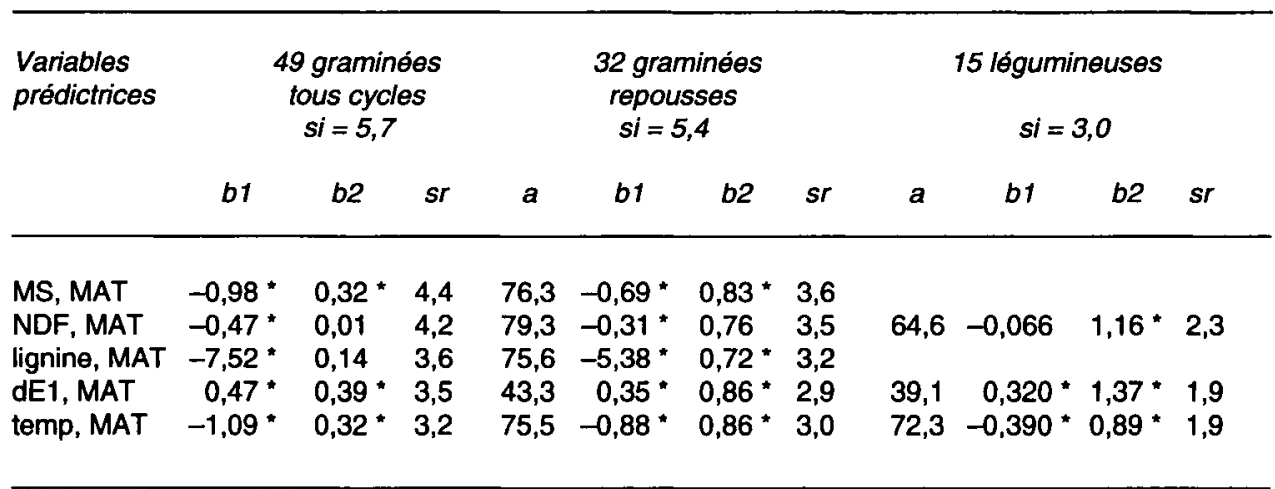

si ou sr : écart type initial ou résiduel; temp : température moyenne pendant la croissance $\left({ }^{\circ} \mathrm{C}\right)$; MAT, lignine et NDF $(\% \mathrm{~ms}) ; \mathrm{MS}(\%)$; dE1 : dégradation enzymatique de l'azote pendant $1 \mathrm{~h}(\% \mathrm{nt}) ;{ }^{*}$ coefficient significatif $(P<0,05)$.

des teneurs en NDF et MAT, obtenue sur repousses de graminées, est assez peu modifiée lorsqu'on élargit la population aux légumineuses tous cycles confondus (tableau VIII). Chez les premiers cycles de graminées, la DTN présente une très bonne liaison linéaire quadratique avec la teneur en MAT (tableau VI). En résumé, la DT augmente avec la teneur en MAT à l'intérieur de populations de fourrages homogènes quant au cycle et aux constituants pariétaux (fig 4).
Sur la population de 72 fourrages, la dE1 est le test de laboratoire le plus lié à la DTN (corrélation de 0,78 contre 0,67 et 0,57 pour $S$ et $F$ respectivement). Sur une population excluant le premier cycle de graminées, la précision s'accroit lorsqu'on associe les MAT à la dE1; elle devient alors supérieure à celle permise par la composition chimique seule (tableaux VII et VIII).

La température moyenne de croissance est la caractéristique des fourrages la plus 
Tableau VIII. Prédiction de la dégradabilité théorique de l'azote (en pourcentage, $k=0,06 \mathrm{~h}^{-1}$ ) des repousses de graminées et des légumineuses $(n=47 ; s i=5,2)$.

$\begin{array}{llllll}\text { Prédictrices } & a & b 1 & \text { b2 } & \text { sr } & R 2\end{array}$

$\begin{array}{lrrrrr}\text { NDF, MAT } & 80,6 & -0,31 & 0,71 & 3,3 & 0,60 \\ \text { dE1, MAT } & 43,2 & 0,41 & 0,74 & 3,0 & 0,67 \\ \text { temp, MAT } & 82,1 & -0,97 & 0,64 & 3,2 & 0,62\end{array}$

si our sr : écart type initial ou résiduel; temp : température moyenne pendant la période de croissance $\left({ }^{\circ} \mathrm{C}\right)$; MAT et NDF (\%ms); dE1 : dégradation enzymatique de l'azote pendant $1 \mathrm{~h}$ (\%nt).

liée à la DTN sur la population complète. À l'intérieur d'une population de fourrages ayant poussé à même température, la DTN augmente avec la teneur en MAT (fig 4). L'association de la température et de la teneur en MAT conduit à une précision toujours supérieure à celle obtenue avec la

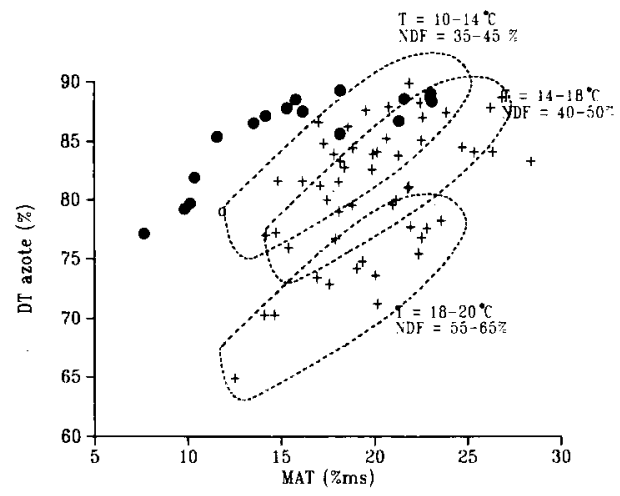

Fig 4. Relation entre la dégradabilité théorique de $N\left(k=0,06 h^{-1}\right)$ et la teneur en MAT; 72 fourrages verts; : 1 er cycle à tiges de graminées; + : autres; $T$ : température moyenne journalière pendant la période de croissance. composition chimique seule (tableaux VII et VIII).

\section{DISCUSSION}

\section{Prédiction de la DTN}

Le rôle significatif des parois a été peu signalé dans les rares études portant sur la prédiction de la DTN des fourrages verts. Sur une population hétérogène, Glenn et al (1985) ont également obtenu une diminution significative de la DTN avec la teneur en NDF, mais pas avec l'ADF. Les parois totales, estimées par le NDF, limitent la dégradation des protéines liées aux parois et de celles des contenus cellulaires, mais seulement en début d'incubation car une fraction importante de ces parois est digérée rapidement. L'ADF. à l'inverse, qui représente des parois plus difficilement dégradables (cellulose + lignine), est associé aux phénomènes de fin d'incubation. Chez les graminées, la teneur en lignine pourrait être un témoin de l'effet plus global de la température sur les parois. Elle traduit également la particularité du dactyle, plus riche en lignine que les autres espèces, et le vieillissement au $1^{\mathrm{er}}$ cycle. II est vraisemblable que l'augmentation de la lignification des parois contribue directement à diminuer l'accessibilité des contenus cellulaires. Cependant, l'amélioration de la précision obtenue avec la lignine est partiellement artificielle. En effet, le fichier des teneurs en lignine avait été épuré de ses valeurs aberrantes (15\%); les risques d'erreur seront toujours présents lors du contrôle sur un échantillon. Il est connu que le dosage de la lignine est une méthode peu précise, d'autant plus que l'extraction préalable au détergent neutre en solubilise une partie va- 
riable chez les graminées (Giger et Pochet, 1987).

La teneur en MS n'agit probablement pas directement sur la dégradabilité de $\mathrm{N}$, mais reflète les variations de la température, qui influe elle-même sur les parois. Sa moindre signification est probablement due à cette action indirecte, mais également aux grandes fluctuations que ce paramètre subit d'un jour à l'autre selon les conditions météorologiques. La teneur en MS présente cependant l'avantage d'être très facile à obtenir.

Nos résultats sont cohérents avec les données de la littérature sur l'influence des MAT (Filmer, 1982; Glenn et al, 1985; Madsen et Hvelplund, 1985; Van Eys et Reid, 1987; Lindberg, 1988; Van Straalen et Tamminga, 1990). II n'y a généralemnt pas de relation entre DTN et MAT quand la population comporte plusieurs espèces et cycles différents. En revanche, à l'intérieur d'une population homogène, la DTN s'accroît de 0,35 à 1,6 point par point de MAT supplémentaire. Chez les graminées, la pente est plus accentuée au premier cycle $(1,5)$ que pour les repousses $(0,5$ à 0,7$)$; et Lindberg (1988) obtient comme nous une courbe à plateau au premier cycle. Seul Ould-Bah (1989), qui travaille sur des résidus de sachets décontaminés des microbes du rumen, a trouvé une excellente relation sur une population limitée mélangeant premier cycle et repousses. Cependant, la DTN obtenue par cet auteur est très bien liée à la DTN prédite par notre équation associant NDF et MAT sur graminées et légumineuses $\left(R^{2}=0,90\right)$. Filmer (1982) a expliqué cet effet de la teneur en azote, indépendant de l'effet des parois, par une accumulation du $\mathrm{N}$ additionnel en partie sous forme soluble ou non protéique dans les contenus cellulaires plutôt que dans les parois. La DT plus élevée des premiers cycles à tiges de graminées, comparée à des graminées feuillues de mêmes teneurs en
NDF et MAT, est cohérente avec la proportion d'azote non protéique plus élevée dans les tiges que dans les feuilles (Demarquilly et al, 1981).

\section{Signification des valeurs de DTN}

La comparaison de nos résultats moyens aux valeurs de dégradation de l'azote des fourrages vert mesurées in vivo présente 2 limites majeures : 1) compte tenu des problèmes méthodologiques liés à sa mesure, la DTN est seulement un indice relatif de la dégradation vraie (Vérité et al, 1987); 2) les estimations in vivo sont peu nombreuses, souvent très variables et entachées d'erreurs relatives à la mesure des flux duodénaux et à l'estimation des fractions microbienne et endogène (Beever, 1982; Thomson, 1982). Vérité et al (1987) ont montré que la DTN surestimait légèrement la dégradation in vivo. Cela pris en compte, notre DTN moyenne ( $82 \%$ ) est cohérente avec la dégradation in vivo moyenne, qui peut être estimée à partir de la revue de Demarquilly et Jarrige (1982) sur les fourrages verts (environ $80 \%$ ). Elle est également cohérente avec celle qui découle des relations de prédiction du flux d'azote duodénal à partir de la teneur en azote et de la matière organique digestible des fourrages verts $(79 \%, n=65$, Peyraud, communication personnelle). Nos résultats sont également en accord avec les teneurs en ammoniac ruminal et les pertes azotées digestives importantes, ainsi que la prédominance de la fraction microbienne dans l'azote duodénal, observées avec les fourrages verts (Beever, 1982; Thomson, 1982).

Sur des populations de fourrages verts, différents auteurs ont obtenu des valeurs moyennes de DTN $\left(k=0,06 \mathrm{~h}^{-1}\right)$ comprises entre 55 et $75 \%$ (Syrjälä-Qvist et al, 1984; Glenn et al, 1985; Madsen et Hvel- 
plund, 1985; Van Eys et Reid, 1987; Lindberg, 1988; Van Straalen et Taminga, 1990). Les différences avec nos résultats peuvent être dues à la plus faible teneur en MAT des fourrages pour ces auteurs, et aux différentes modalités d'application de la méthode des sachets de nylon (Oldham, 1987). Le conditionnement des fourrages est particulièrement important : il apparaît nettement que les fourrages séchés à l'étuve ou hachés grossièrement en frais ont une DTN inférieure à celle des fourrages lyophilisés. II est par ailleurs probable que les variations de DTN que nous observons soient partiellement dues à la contamination microbienne des sachets. En effet, le couple NDF-MAT intervient avec des coefficients de même signe dans notre prédiction de la DTN, et dans la sous-estimation de DTN induite par la contamination, évaluée par MichaletDoreau et Ould-Bah (1989).

\section{CONCLUSION}

Alors que le stade, l'espèce, la saison, la fertilisation azotée et le lieu de récolte ont séparément une influence modérée sur la DT de l'azote des fourrages verts, le cumul de leurs effets se traduit par des differences de DT importantes entre fourrages extrêmes de la population. Elles s'expliquent partiellement par des différences de conditions de croissance et de composition chimique. Dans une population hétérogène, la DTN est avant tout liée négativement à la température et aux parois; dans une population homogène en cycle, température et teneur en parois, elle augmente avec la teneur en MAT. Ces observations demandent à être mieux expliquées et confirmées : rôle de la température sur la physiologie de la plante, influence de la teneur en MAT sur la répartition des différentes fractions protéiques et non protéiques. La prédiction de la DTN est réalisable en pratique à partir de la seule composition chimique simple, mais la précision est très améliorée lorsque la $\mathrm{dE} 1$ est introduite dans les équations.

L'hypothèse d'un rythme d'évacuation des particules hors du rumen constant, quel que soit le fourrage $\left(k=0,06 \mathrm{~h}^{-1}\right)$ a probablement conduit à augmenter les différences de DTN, car $k$ varie dans le même sens que la vitesse de digestion des fourrages. Une meilleure connaissance des facteurs de variation du transit des fourrages verts permettrait d'éclaircir ce point. Enfin, il semble inévitable d'intensifier les mesures in vivo des variations de l'apport protéique des fourrages verts; elles devraient permettre : 1) d'étalonner la dégradabilité en sachets et confirmer les variations de la valeur PDIA; 2) de préciser les variations de la synthèse microbienne, dont la contribution à l'azote duodénal est prédominante.

\section{REMERCIEMENTS}

Nous adressons nos sincères remerciements à l'ensemble du personnel (installations expérimentales, laboratoire et secrétariat) de la station INRA de recherches sur la vache laitière.

\section{RÉFÉRENCES}

ADDAD (1989) Manuel de référence. Version micro Addad, Paris

Alderman (1987) Comparison of rations calculated in the different systems. In: Feed evaluation and protein requirement systems for $\mathrm{ru}$ minants (Jarrige R, Alderman $G$, eds). CEC L, Luxembourg 283-298

Aufrère J, Cartailler D (1988) Mise au point d'une méthode de laboratoire de prévision de la dégradabilité des protéines alimentaires des aliments concentrés dans le rumen. Ann Zootech 37, 255-270 
Beever DE (1982) Protein utilization from pasture. In: Forage protein conservation and utilization (Griffith TW, Maguire MF, eds). CEC, Dublin, 99-119

Demarquilly C, Grenet E, Andrieu J (1981) Les constituants azotés des fourrages et la prévision de la valeur azotée des fourrages. $\boldsymbol{n}$ : Prévision de la valeur nutritive des aliments des ruminants. INRA, Versailles, 129-154

Demarquilly C, Jarrige R (1982) Assessment of the protein value of forages and its expression in the new protein feeding systems. In: Forage protein conservation and utilization (Griffith TW, Maguire MF, eds). CEC, Dublin, 147-173

Escofier B, Pagès J (1988) Analyses factorielles simples et multiples objectifs, méthodes et interprétation. Dunod, Paris

Filmer DG (1982) Assessment of protein degradability of forage. In: Forage protein in ruminant animal production (Thomson DJ, Beever DE, Gunn RG, eds). Br Soc Anim Prod OCC publ 6, Haddington, 129-138

Giger S, Pochet S (1987) Méthodes d'estimation des constituants pariétaux dans les aliments destinés aux ruminants. Bull Tech CRZV Theix INRA 70, 49-60

Glenn BP, Ely DG, Glenn S, Douglas LW, Bull LS, Bish LP (1985) Effects of ammonium nitrate and potassium sulfate fertilization on rates of ruminal in situ disappearance of tall fescue and orchard grass nitrogen and sulfur. Can J Anim Sci 65, 631-646

Le Goffe P (1988) Évolution de la dégradabilité de l'azote des fourrages verts au cours du 1 er cycle de végétation. Reprod Nutr Dév 28, 165-166

Le Goffe P (1991) Méthodes d'étude et facteurs de variation de la dégradabilité de l'azote des fourrages verts dans le rumen. Thèse de I'École nationale supérieure agronomique de Rennes, $82 \mathrm{p}$

Le Goffe P, Vérité R (1992) Évolution de la dégradabilité de l'azote du ray-grass anglais au cours de la saison de pâturage. Ann Zootech $41,39-40$

Le Goffe P, Vérité R, Lemaire G (1989) Influence du niveau de fertilisation azotée sur la dégradabilité de l'azote de la fétuque élevée récoltée en vert. In: XVI Intemational Grassland Congress, Nice, France, 911-912
Le Goffe P, Vérité R, Peyraud JL (1993) Influence de l'espèce et de la saison sur la dégradabilité de l'azote des fourrages verts dans le rumen. Ann Zootech 42,1

Lindberg JE (1988) Influence of cutting time and $\mathrm{N}$ fertilization on the nutritive value of timothy. II. Estimates of rumen degradability of nitrogenous compounds. Swed J Agric Res 18, 85-89

Madsen J, Hvelplund T (1985) Protein degradation in the rumen. A comparison between in vivo nylon bag, in vitro and buffer measurements. Acta Agric Scand suppl 25, 103-124

Michalet-Doreau B, Ould-Bah MY (1989) Estimation of the extent of bacterial contamination in bag residues and its influence on in sacco measurements of forage nitrogen degradation in rumen. In: XVI International Grassland Congress, Nice, France, 909-910

Oldham JD (1987) Testing and implementing the modern systems: UK. In: Feed evaluation and protein requirement systems for ruminants (Jarrige R, Alderman G, eds). CEC L, Luxembourg, 269-281

Ould-Bah MY (1989) Adaptation de la technique in sacco à l'étude de la dégradation dans le rumen de l'azote des fourrages et application à l'étude des fourrages verts et conservés. Thèse de l'Université de Montpellier, $186 \mathrm{p}$

Syrjälä-Qvist L, Pekkarinen E, Setälä .J, Kangasmäki $T$ (1984) Effect of nitrogen fertilization on the protein quality of timothy grass and silage. J Agric Sci Finl 56, 193-198

Thomson DJ (1982) The nitrogen supplied by the supplementation of fresh or grazed torage. In: Forage protein in ruminant animal production. Br Soc Anim Prod 6, 53-66

Van Eys JE, Reid RL (1987) Ruminal solubility of nitrogen and minerals from fescue and fescue-red clover herbage. J Anim Sci 65, 11011112

Van Soest PJ (1963) Use of detergents in the analysis of fibrous feeds. II. A rapid method for the determination of fiber and lignin. $J$ Assoc Off Anal Chem 46, 829-835

Van Soest PJ, Wine RH (1967) Use of detergents in the analysis of fibrous feeds. Determination of plant cell-wall constituents. $J$ Assoc Off Agric Chem 50, 50-55 
Van Straalen WM, Tamminga S (1990) Protein degradation of ruminant diets. In: Feedstuff evaluation (Wiseman J, Cole DJA, eds) Butterwork, London, 55

Vérité R, Demarquilly C (1978) Qualité des matières azotées des aliments pour ruminants.
In : La vache laitière. INRA, Versailles, 143157

Vérité R, Michalet-Doreau B, Chapoutot $P$, Peyraud JL, Poncet $C$ (1987) Révision du système des protéines digestibles dans l'intesän (PDI). Bull Tech CRZV Theix INRA 70, 19-34 